\title{
Video Based Adult and Child Classification by Using Body Proportion
}

\author{
Omer F. Ince, M. Eren Yildirim, J.S. Park, J.Song, B.W. Yoon \\ Department of Electronics Engineering, Kyungsung University, Busan, South Korea
}

Keywords: haar-like feature, biometric evaluation, adult and child classification.

\begin{abstract}
Pedestrian detection in uncontrolled environments is a challenging task. There are various researches for video based pedestrian detection. Moreover, to recognize children and adults in digital platforms is helpful for future applications. For instance, if a Closed Circuit Television(CCTV) camera located on a traffic light detects child who is walking through pedestrian way, system could make some service adjustments. Aim of this article is to detect children and adults separately. We used Haar cascade classifiers for the implementation. We detected head and body of pedestrians. Then we used relative measurements because we cannot get exact height of people from pixels so we applied another method that is proportioning head size to body size of pedestrians. By this technique, we could discriminate children and adults. The results are promising and shows sufficient accuracy.
\end{abstract}

\section{Introduction}

With growing demands and technical facilities in image processing area, researches have increased. Object detection and classification are essential and important subtopics of image processing technology. By using object detection algorithms, certain targets such as pedestrians, vehicles, or subsets like gender and age recognition can be done in still images and videos. A wide range of object detection applications in various areas of computer vision had been done.

The main purpose of object detection is to find the location of target in a scene. It is required to be impeccable and fast in detecting objects and understanding situations with video processing. One of the most significant stages of object detection is feature selection. To make impeccable real time object detection, features need to be robust, differential, and easy to calculate [1].

As well as grownups, children also have an active role in society. Unlike grownups, kids do not pay attention to vehicles nor the other people on the street. On the other hand they should be more careful than adults in traffic. Another motivation for this study is to be used for creating data set in the field of health, sociology, traffic etc [2].

Recognizing adults and children is a subclass of pedestrian detection. For that purpose, various effective techniques for age recognizing are represented.

One of these techniques is ratio of head to body size. It is medical fact that with increasing age, head height of a human becomes relatively much smaller compared to the body height. Starting from this point of view a new method can be developed.

In this paper, we describe classification of people as adult or child by using ratio of head and body heights. Main frame of our work is detecting pedestrians in a scene and proportion their heights of head to body. The decision is made according to the predefined ratio value.

\section{Adaboost algorithm and Haar-like feature based detection}

\section{Detection based on Haar-like feature}

It is introduced by Viola and Jones [3], for rapid object detection and is applied to the pedestrian detection. With the simple haar-like features which can be calculated efficiently by using integral images and Adaboost classifiers in a cascade structure, their detector has high detection speed [4]. Experiments showed that object detection using Haar-like features can achieve high accuracy at a considerably low cost. 
Two or three adjacent rectangular groups with different contrast values create Haar-like features[5]. The intensity values of white and black groups are separately calculated and subtracted from each other. This subtraction will give light and dark regions. It is essential to say that the concept of lightness and darkness here is relative. Fig.1 shows a set of Haar-like features. These rectangles can be rotated to increase their variety.

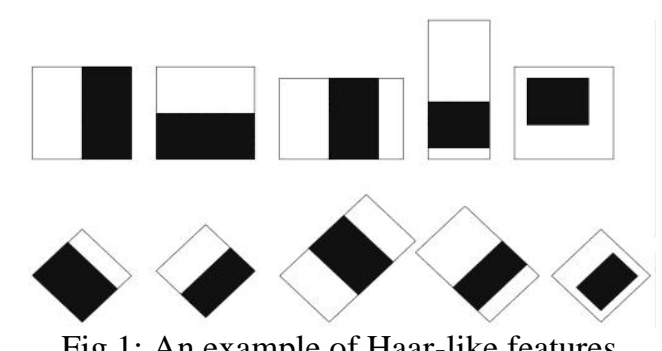

Fig.1: An example of Haar-like features

\section{Adaboost algorithm}

In general, Boosting is a statistical method that uses the original distribution of positive and negative examples to compute simple rules also called weak classifiers and combines them to create a stronger classifier. AdaBoost is most commonly used for binary classification, but with minor modifications it can also deal with multiple classes [6].

\section{Proposed Algorithm}

In this part, proposed algorithm which is used to recognize children and adults in videos is given. By using Haar-like features, recognition of age is very difficult when the capture is done from a far distance. Key point of this study is to use biometric ratios instead of steady values. Implementation steps are shown in Fig. 2. The algorithm consists of several steps, but it can be divided into two main parts.

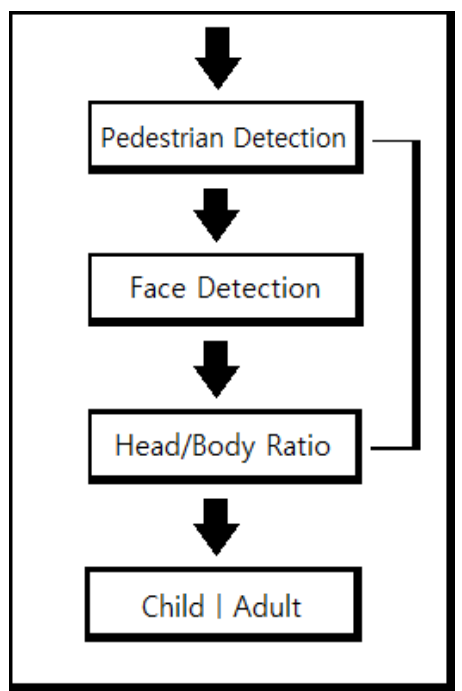

Fig. 2: Block diagram of the detecting adults and children in digital images

In a still image captured from our test video, there are various objects. To detect the pedestrian and pedestrian's head in it, we use Haar cascade detector which we created previously.

First step is to detect the full body. After we detect a body, our new region of interest would be pedestrian body. So, we need to detect head of that specific pedestrian. In this stage we use Haar cascade detector that is was trained to detect head. By doing these steps, we finished first two steps which is shown in block diagram.

For third step, we need to calculate head and body ratio by equation (1). 


$$
r=\frac{l_{H}}{l_{B}}, 0<r<1
$$

$l_{B}$ and $l_{H}$ are heights of body and head, respectively. $r$ means the ratio of $l_{B}$ and $l_{H}$.

The main reason of this calculation is that people's head height becomes relatively smaller than body height as they grow up. In still images, we cannot measure absolute value of people's heights from pixels. That's why if we apply relative measurement which is related to proportioning head to body height, problem would be solved. By this idea, we can classify pedestrian as child or adult. Our decision rule is as given in (2). If head over body ratio is smaller than 0.3 , we can then classify that pedestrian as an adult. If that ratio is equal or larger than 0.3 , it means the pedestrian is child.

$$
\text { Output }=\left\{\begin{array}{lll}
\text { Adult } & \text { if } & r<0.3 \\
\text { Child } & \text { if } & r \geq 0.3
\end{array}\right\}
$$

\section{Experiments and Results}

In training step we used images from the INRIA person dataset and CVC-CER-01(Computer Vision Center) pedestrian dataset [7]. Training and all tests are done in a $2.60 \mathrm{GHz}$ computer by using Microsoft Visual Studio 2010 and OpenCV2.2. The test videos are recorded by CCTV cameras located on street and subway stations in Korea.

In output images, adult pedestrians were marked with red rectangular and children were marked as blue rectangular. Rectangular is located on the center of pedestrians face. Green rectangular represents pedestrian body. There were some false positives and true negatives observed in certain images but they are tolerable.

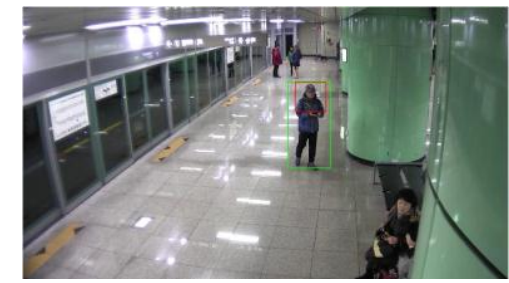

(a)

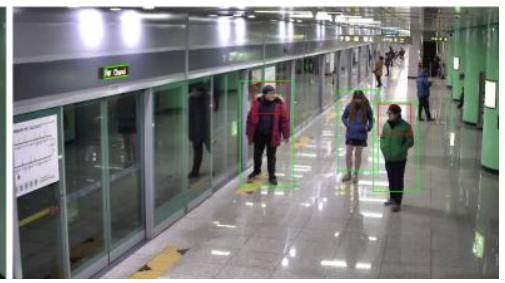

(b)

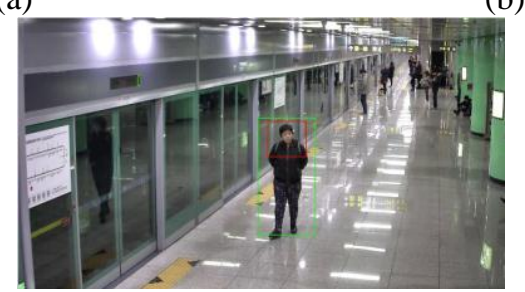

(c)

Fig.3: Captures of results for detecting adults

Figure 3 shows successful classifying results for adults. Only error appeared in (b), as the girl is not decided as an adult.

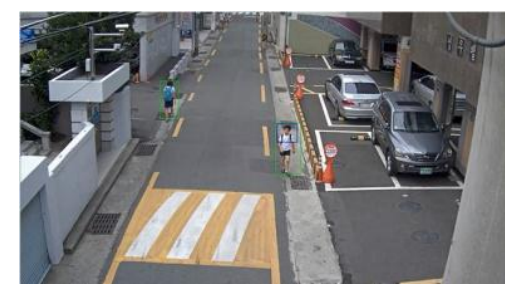

(a)

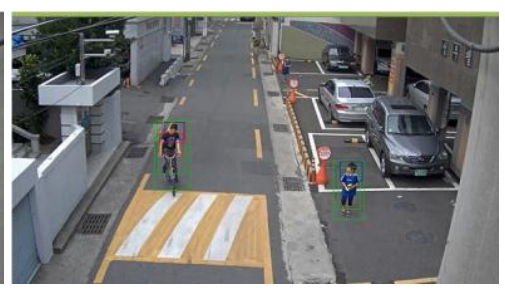

(b) 


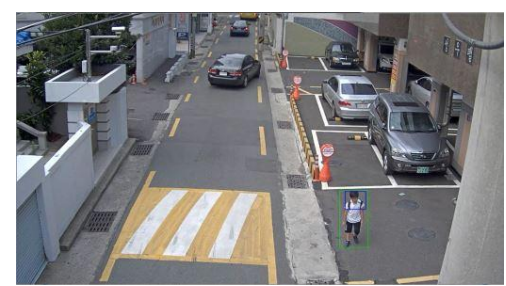

(c)

Fig.4: Captures of results for detecting children

Figure 4 shows the results for children recognition. In (b) and (c), error occurred for the kids on the left side. One of them is gives a false positive where as the other one is a true negative.

\section{Conclusion}

In this paper, a new approach is proposed to classify adult and child on videos. We showed a new method which is related to relative measurement for classifying problem. Absolute measurement cannot be done in digital images, so we concentrate on relative measurement. Although it can not exactly estimate heights of pedestrian, it showed that this approach can classify adult and child effectively. In the future study, we will focus on performance and accuracy enhancement.

\section{Acknowledgement}

This work was supported by the Industrial Strategic technology development program of Ministry of Science, ICT and Future Planning, Korea, (10045260, Development of Context Awareness Monitoring and Search System Based on High Definition Multi-video) and Brain Busan, Busan City.

\section{References}

[1] K. Lee, C.H. Lee, S.A. Kim, Y.H. Kim, Fast Object Detection Based on Color Histograms and Local Binary Patterns. TENCON IEEE Region 10 Conference, Cebu, Nov. 19-22, 2012.

[2] H. Weda, M. Barbieri, Automatic Children Detection in Digital Images. IEEE International Conference on Multimedia and Expo, Beijing, July. 2-5, 2007.

[3] P. Viola and M. Jones. Rapid object detection using a boosted cascade of simple features. Proceedings of the 2001 IEEE Computer Society Conference on Computer Vision and Pattern Recognition, vol.1, pp. 511-518, 2001.

[4] G. R. Rakate, S. R. Borhade, P. S. Jadhav, M. S. Shah. Advanced Pedestrian Detection System Using Combination of Haar-like Features, Adaboost Algorithm and Edgelet-Shapelet, IEEE Conference on Computational Intelligence and Computing Research, Coimbatore, India, December 18-20, 2012.

[5] Mustafa E. Yildirim, J. S. Park, J. Song, and B. W. Yoon. Gender Classification Based on Binary Haar Cascade. International Journal of Computer and Communication Engineering, Vol. 3, No. 2, March 2014.

[6] V. Viola and M.J. Jones. Robust Real-Time Face Detection, International Journal of Computer Vision, Vol.57, pp.137-154, 2004.

[7] D. Geronimo, A.D. Sappa, A. Lopez and D. Ponsa. Adaptive Image Sampling and Windows Classification for On-Board Pedestrian Detection, Proceedings of the International Conference on Computer Vision Systems, Bielefeld, Germany, March, 2007. 